\title{
Clinical utility of azilsartan-chlorthalidone fixed combination in the management of hypertension
}

\author{
This article was published in the following Dove Press journal: \\ Vascular Health and Risk Management \\ 12 June 2012 \\ Number of times this article has been viewed
}

\author{
Jerrica E Shuster ${ }^{1,2}$ \\ Barry E Bleske ${ }^{1,2}$ \\ Michael P Dorsch ${ }^{1,2}$ \\ 'University of Michigan Hospitals \\ and Health Centers, Ann Arbor, MI, \\ USA ${ }^{2}$ College of Pharmacy, University \\ of Michigan, Ann Arbor, MI, USA
}

\begin{abstract}
Azilsartan-chlorthalidone fixed combination is a new drug in the management of hypertension. Azilsartan has been shown to have greater blood pressure-lowering effects than other angiotensin-receptor blockers (ARBs), and the debate regarding the superiority of chlorthalidone over hydrochlorothiazide has been ongoing for years. The combination is unique because it is the first to partner an ARB with this, possibly more effective, diuretic. This review will address trials involving both components of this drug, as well as phase III trials involving the fixed-combination product. The article will also discuss the benefit of combination therapy in the treatment of hypertension.
\end{abstract}

Keywords: azilsartan, chlorthalidone, combination, hypertension

\section{Introduction}

Despite the knowledge gained from large clinical trials, cardiovascular disease remains one of the leading causes of death, and hypertension remains a significant cause of cardiovascular morbidity and mortality. According to the National Heart, Lung, and Blood Institute, over one-third of the United States was diagnosed with hypertension during 2005-2008. Fortunately, over 70\% of these patients were being treated for their hypertension, but disappointingly only $47.7 \%$ had controlled blood pressure (BP). ${ }^{1}$ This lack of medical success is one reason why new antihypertensive agents continue to be developed and also explains the interest in fixed-combination antihypertensive agents, such as the new angiotensin-receptor blocker/thiazide-type combination of azilsartan-chlorthalidone.

The current guidelines recommend administering more than one drug as initial therapy for BP $>20 \mathrm{mmHg}$ systolic or $>10 \mathrm{mmHg}$ diastolic above goal, with one of these drugs being a thiazide-type diuretic. ${ }^{2}$ Angiotensin-receptor blockers (ARBs) as initial therapy have also fallen into favor due to their cardiovascular morbidity and tolerability benefits over beta blockers. ${ }^{3}$ Several trials have shown that combination therapy increases achievement of BP control at lower doses which also decreases side effects. ${ }^{4-6}$ When using combination therapy, fixed-combination agents have the benefit of also increasing efficacy by increasing compliance. ${ }^{4}$

Several fixed-combination antihypertensive agents exist, and seven of them are prescribed enough to be on the top 200 drugs list. ${ }^{7}$ Azilsartan-chlorthalidone is unique in that it is the only combination drug that contains an ARB with the long-acting thiazide-type diuretic, chlorthalidone. ${ }^{8}$ This article will discuss the clinical utility of fixed-combination azilsartan-chlorthalidone in the management of hypertension.
Correspondence: Jerrica Shuster I500 E Medical Center Dr, SPC 5008, Ann Arbor, MI 48109, USA

Tel +l 7349368210

Fax + I 7349367027

Email jerricas@med.umich.edu 


\section{Azilsartan: a new ARB}

Angiotensin II (AngII) is an integral hormone in the regulation of BP. AngII causes most of its hypertensive effects by stimulating the angiotensin type I receptor (AT1), making this receptor the primary target of ARBs. When activated, AT1 results in hypertension from direct vasoconstriction and secretion of aldosterone, and central AngII has also been shown to affect regulation of the sympathetic nervous system. ${ }^{9}$ ARBs have shown their value in BP therapy by being as efficacious as angiotensin-converting enzyme (ACE) inhibitors, while causing fewer side effects than other drug classes, including idiosyncratic cough and angioedema associated with ACE inhibitors. ${ }^{10}$ Within the ARB class, there are differences in pharmacokinetic and pharmacologic properties that may translate into different therapeutic effects, including BP-lowering.

The newer ARB, azilsartan, is commercially available as $40 \mathrm{mg}$ and $80 \mathrm{mg}$ oral tablets. The bioavailability of the drug is estimated at $60 \%$, with peak plasma concentrations obtained at 1.5 to 3 hours. Azilsartan demonstrated a volume of distribution of approximately $16 \mathrm{~L}$ and is highly protein-bound ( $>99 \%$ ). The half-life has been measured at 11 hours, and the major enzyme responsible for its metabolism is CYP2C $9 .{ }^{11}$

Azilsartan, a prodrug, is a selective and insurmountable AT1 antagonist with evidence suggesting it not only has high binding affinity, but also has slow dissociation from the target receptor. ${ }^{12}$ Azilsartan has been shown in vitro to have a higher percentage of inhibition at human AT1 than olmesartan, telmisartan, valsartan, and irbesartan. In the same study, azilsartan also showed the greatest receptor binding after washout when compared to the other ARBs. ${ }^{12}$ These data suggest that azilsartan may bind more tightly and dissociate more slowly from the selective target AT1 than the comparator ARBs. Clinically, azilsartan's binding characteristics may allow for this drug to have greater BP-lowering effects and a longer duration of action compared to other ARBs.

The insurmountable, or tight-binding behavior, may be attributable to the presence of a carboxyl group as part of the ARB chemical structure. The ARBs with a carboxyl moiety and insurmountable binding include olmesartan, candesartan, and azilsartan. ${ }^{12}$ The carboxyl group is thought to be responsible for interaction with the amino acid lysine and provide tight binding to AT1. The carboxyl group may also be related to the beneficial decrease in hypertrophy exhibited by these ARBs. In addition to a carboxyl group, azilsartan also has a distinctive oxadiazolone group in place of the tetrazole ring that exists as part of the structure on other currently available ARBs. It is possible that the oxadiazolone structure in azilsartan allows azilsartan to bind more strongly to essential residues of the AT1. Tighter receptor binding, as has been shown with azilsartan, may be an advantageous characteristic since this binding would allow azilsartan to maintain its effects even when AngII levels increase. ${ }^{12}$ When ARBs are administered to patients, AngII levels are known to increase as a compensatory mechanism caused by AT1 inhibition during ARB treatment. Elevated AngII levels may theoretically displace ARBs from their receptor sites. ARBs with tighter binding characteristics may be less likely to be displaced resulting in greater therapeutic efficacy as compared to ARBs with less tight binding characteristics. Azilsartan's unique structure and pharmacological properties may provide advantages in the treatment of hypertension compared to other ARBs.

\section{Azilsartan versus other ARBs}

As a monotherapy, azilsartan $80 \mathrm{mg}$ once daily has been shown to be more effective in lowering systolic and trough $\mathrm{BP}$ than other ARBs at their highest approved doses. One trial compared increasing doses of azilsartan $(20,40$, and $80 \mathrm{mg}$ ) to olmesartan $40 \mathrm{mg}$ and placebo. ${ }^{13}$ The primary endpoint in this trial was a change in 24-hour mean ambulatory BP after 6 weeks of treatment in 1260 randomized essential hypertension patients. Clinic BP (measured 24 hours after previous dose) and percentage of responding patients were evaluated in this study. A "responder" was defined as a patient achieving systolic BP (SBP) of $<140 \mathrm{mmHg}$ or a decrease of $\geq 20 \mathrm{mmHg}$. All BP was measured with an automated device. The trial excluded patients with estimated glomerular filtration rate (eGFR) $<30 \mathrm{~mL} / \mathrm{min}$ and type I or poorly controlled type II diabetes. The $40 \mathrm{mg}$ dose of azilsartan was shown to be noninferior to olmesartan. The difference of change in 24-hour mean SBP was $-2.1 \mathrm{mmHg}$ (95\% confidence interval [CI]: $-4.0-0.1 ; P=0.038$ ) greater with azilsartan $80 \mathrm{mg}$ compared to olmesartan $40 \mathrm{mg}$. The results also showed a $-2.7 \mathrm{mmHg}$ greater decrease $(95 \%$ CI: $-5.3-0.1 ; P=0.043)$ in clinical SBP with administration of azilsartan $80 \mathrm{mg}$ compared to olmesartan. However, there was no significant difference in the amount of patients that achieved clinical response (57\% azilsartan and 53\% olmesartan; $P=0.402$ ) between these two arms. Although not statistically significant, a subgroup analysis regarding patients with a body mass index $(\mathrm{BMI}) \geq 30 \mathrm{~kg} / \mathrm{m}^{2}$ showed a treatment difference between azilsartan $80 \mathrm{mg}$ and olmesartan $40 \mathrm{mg}$ of $-2.7 \mathrm{mmHg}(95 \% \mathrm{CI}:-5.8-0.32$ ) compared to a $-1.7 \mathrm{mmHg}$ difference in patients with a BMI $<30(95 \%$ CI: -4.2-0.9). There was no difference in adverse effects between all treatment groups. In conclusion, azilsartan 
showed a statistically significant decrease in SBP based on 24-hour ambulatory BP and trough (clinical) BP.

A trial similar in patient population, timeline, and primary outcome to the previously discussed trial above, compared azilsartan $40 \mathrm{mg}$ and $80 \mathrm{mg}$ to placebo, olmesartan $40 \mathrm{mg}$, or valsartan $320 \mathrm{mg}$ in 1175 randomized hypertensive patients. ${ }^{13,14}$ This study assessed the same endpoints of mean 24-hour BP, clinical BP, and percentage of responding patients. All BP measurements were obtained using automated devices. Comparable to the previous trial, azilsartan $40 \mathrm{mg}$ was shown to be noninferior to olmesartan, while azilsar$\tan 80 \mathrm{mg}$ showed superiority to both of the highest doses approved for olmesartan and valsartan (difference in reduction of mean 24-hour SBP $-2.5 \mathrm{mmHg}, 95 \% \mathrm{CI}$ : -4.4-0.6; $-4.3 \mathrm{mmHg}$, 95\% CI: $-6.3-2.4$, respectively). Azilsartan $40 \mathrm{mg}$ and $80 \mathrm{mg}$ also had a greater reduction in clinical BP compared to olmesartan $40 \mathrm{mg}$ and valsartan $320 \mathrm{mg}(-14.6$, $-14.9,-11.4$, and $-9.5 \mathrm{mmHg}$, respectively; all $P<0.05)$. Unlike the previous trial, this trial found a significantly larger amount of "responders" in the azilsartan arm (58\%) than the valsartan $(49 \%)$ or olmesartan $(49 \%)$ arms $(P<0.05)$. No difference was seen when comparing obese to nonobese patients, and all treatment arms showed similar adverse events.

The above trials demonstrated that azilsartan has greater efficacy in decreasing overall SBP and greater BP effects at the end of the dosing interval. The extent of azilsartan's effects on SBP, although marginal, may prove to be clinically significant as current guidelines discuss a decrease of cardiovascular events with SBP reductions of $2 \mathrm{mmHg}{ }^{2}$
In addition, it should be emphasized that azilsartan actually showed superiority to agents in the same class as opposed to merely demonstrating noninferiority. These findings suggest azilsartan could be an innovative ARB that has distinct advantages over the other ARBs and these advantages may be related to its pharmacological profile.

\section{Azilsartan-chlorthalidone combination}

The combination of azilsartan-chlorthalidone is a novel combination agent because it is the first to combine an ARB with the long-acting diuretic, chlorthalidone. This fixed-dose combination (Edarbyclor ${ }^{\circledR}$; Takeda Pharmaceutical, Osaka, Japan) was recently approved by the US Food and Drug Administration (FDA) on December 20, 2011 as 40/12.5 mg and 40/25 mg dosages. The first phase III trial to evaluate this combination was a randomized, double-blind, multicenter, 6-week treatment study comparing two different doses of azilsartan (40 mg or $80 \mathrm{mg}$ ) combined with $25 \mathrm{mg}$ chlorthalidone to $25 \mathrm{mg}$ chlorthalidone monotherapy in essential hypertension patients. ${ }^{15}$ Baseline characteristics were not reported in the study. The results showed a statistical decrease in 24-hour mean SBP in both the azilsartan-chlorthalidone 40/25 $\mathrm{mg}$ and 80/25 $\mathrm{mg}$ arms (-31.72 and $-31.3 \mathrm{mmHg}$, respectively; $P<0.001)$ when compared to chlorthalidone alone $(-15.85 \mathrm{mmHg})$. Similar results were seen when comparing mean diastolic pressure, mean daytime systolic pressure, and mean nighttime systolic pressure (Figure 1). Another secondary outcome examined the difference

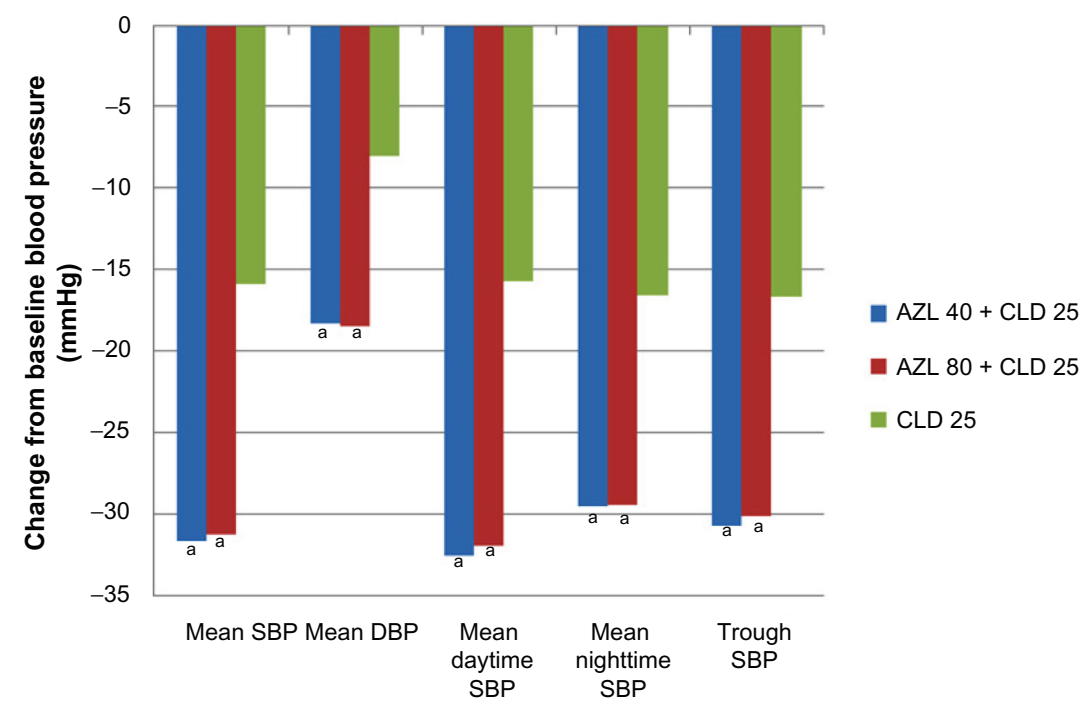

Figure I Change from baseline blood pressure measures at 6 weeks.

Notes: a $P<0.001$ compared to chlorthalidone $25 \mathrm{mg}$ daily, mean daytime systolic blood pressure was measured from 6 am to 10 pm, mean nighttime systolic blood pressure was measured from 12 am to 6 am, trough SBP was measured 22 to 24 hours after the dose was given. ${ }^{15}$

Abbreviations: AZL, azilsartan; CLD, chlorthalidone; DBP, diastolic blood pressure; SBP, systolic blood pressure. 
between each treatment arm regarding trough SBP, defined as systolic measurement recorded 22 to 24 hours after dosing. The difference in trough SBP was statistically greater for both azilsartan combination arms compared to the chlorthalidone arm (40 mg: $-14.04 \mathrm{mmHg}, 95 \% \mathrm{CI}:-17.13-10.94$; $80 \mathrm{mg}$ : $-13.48 \mathrm{mmHg}$, 95\% CI: $-16.58-10.37 ; P<0.001$, respectively). This trial displays that the combination of azilsartan-chlorthalidone is more effective at decreasing BP than chlorthalidone monotherapy. This study also demonstrates azilsartan-chlorthalidone's long-acting benefit by assessing the continued decrease in BP 22 to 24 hours after dosing. This trial did not show any statistical difference in BP effects between the different dosing regimens of azilsartan 40 or $80 \mathrm{mg}$ plus chlorthalidone.

In another trial only published in abstract form, several different fixed-dose azilsartan-chlorthalidone combinations were compared to different doses of both of the monotherapies. This was an 8-week, randomized, double-blind trial in 1714 hypertensive patients with a primary endpoint of change in trough SBP. ${ }^{16}$ Patients received either azilsartan placebo, 20,40 , or $80 \mathrm{mg}$ and chlorthalidone placebo, 12.5, or $25 \mathrm{mg}$ either as monotherapy or in combination. There was no double-placebo arm. Baseline characteristics provided were mean age 57 years, $47 \%$ men, and $20 \%$ black patients, with no differences reported among the treatment groups. All six of the combination therapy arms resulted in a significantly greater decrease in trough SBP when compared to their comparative monotherapy components (Table 1). As seen in Table 1, there was no greater benefit seen when comparing azilsartan-chlorthalidone $40 / 25 \mathrm{mg}$ to $80 / 25 \mathrm{mg}$. There was a dose-dependent increase in therapy discontinuation and serum creatinine seen with azilsartan-chlorthalidone. Interestingly, it was noted in the study that the addition of azilsartan seemed to attenuate the chlorthalidone-related hypokalemia, but no data was provided in the abstract. ${ }^{16}$

Another published abstract examined the fixed-dose combination of azilsartan-chlorthalidone $20 / 12.5 \mathrm{mg}$ titrated to 40/25 mg (AZL40-CLD25), 40/12.5 mg titrated to 80/25 mg (AZL80-CLD25), and olmesartan-hydrochlorothiazide
20/12.5 mg titrated to 40/25 mg (OLM40-HCTZ25) in 1085 patients with hypertension. ${ }^{17}$ Patients were only titrated to the higher dose if their SBP was not at goal by week 4 . This was an 8-week, randomized, double-blind trial. Baseline characteristics included 52\% men, $26.7 \%$ blacks, $17.3 \%$ diabetics, a mean age of 56 years, and a BMI of $31.8 \mathrm{~kg} / \mathrm{m}^{2}$. Both azilsartan-chlorthalidone combinations decreased clinical BP (seated trough BP) significantly more than olmesartan/hydrochlorothiazide (AZL40-CLD25 $=-33.0$ and AZL80-CLD25 = -34.1 vs OLM40-HCTZ25 = -26.9 mmHg; $P<0.001)$. A greater reduction in 24-hour mean SBP was also seen with both azilsartan-chlorthalidone combinations $(-26.4$ and -27.9 versus $-20.7 \mathrm{mmHg} ; P<0.001)$ Similarly, there was also less titration to higher doses in the azilsartan-chlorthalidone arms (38.4\% and 34.7\%) than in the olmesartan/hydrochlorothiazide group (51.7\%). Adverse events causing either temporary or permanent discontinuation of therapy occurred in $6.2 \%$ and $9.5 \%$ of the azilsartan-chlorthalidone arms and $3.1 \%$ of the olmesartan-hydrochlorothiazide group (no $P$ values were provided in the abstract). ${ }^{17}$ These trials demonstrate a greater decrease in mean and trough SBP with the azilsartan-chlorthalidone combination.

\section{Chlorthalidone versus hydrochlorothiazide}

Current guidelines suggest thiazide-type diuretics as first-line treatment in hypertension and list several options, but do not distinguish preference between any agents. ${ }^{2}$ Generally chlorthalidone and hydrochlorothiazide are viewed as interchangeable, but their effects on cardiovascular events have never been compared in a prospective trial. Most of the major clinical trials involving a thiazide-type diuretic have included either chlorthalidone or hydrochlorothiazide, yet hydrochlorothiazide is much more commonly prescribed and included in combination products. ${ }^{18}$ The debate regarding possible differences in clinical benefit between the two agents has been an increasing topic of interest.

Though several differences between the drugs are known, it has not been determined which, or if any, could

Table I Change in trough SBP of combinations when compared to the monotherapy components at week 8

\begin{tabular}{lllll}
\hline LSM SBP reduction $(\mathbf{m m H g})$ & No azilsartan & Azilsartan $\mathbf{2 0} \mathbf{~ m g}$ & Azilsartan $\mathbf{4 0} \mathbf{~ m g}$ & Azilsartan 80 mg \\
\hline No chlorthalidone & - & -12.1 & -12.8 & -15.1 \\
Chlorthalidone $12.5 \mathrm{mg}$ & -12.7 & $-22.9^{\mathrm{a}, \mathrm{b}}$ & $-24.4^{\mathrm{a}, \mathrm{b}}$ & $-26.3^{\mathrm{a}, \mathrm{b}}$ \\
Chlorthalidone $25 \mathrm{mg}$ & -15.9 & $-26.3^{\mathrm{a}, \mathrm{b}}$ & $-29.8^{\mathrm{a}, \mathrm{b}}$ & $-28.0^{\mathrm{a}, \mathrm{b}}$ \\
\hline
\end{tabular}

Notes: a $<<0.001$ compared to chlorthalidone component; ${ }^{b} P<0.001$ compared to the azilsartan component. Copyright $\odot 201 \mathrm{I}$. Reprinted with permission from John Wiley and Sons. American Society of Hypertension. Twenty-Sixth Annual Scientific Meeting and Exposition [featured posters, poster 182]. J Clin Hypertens. 201 1;13:12-163. Available from: http://onlinelibrary.wiley.com/doi/I0.1 III/j.175I-7176.20I I.00459.x/abstract. Accessed January 2011. ${ }^{16}$

Abbreviations: LSM, least squares mean; SBP, systolic blood pressure. 
cause a change in patient outcomes. Structural differences exist between chlorthalidone and hydrochlorothiazide. Hydrochlorothiazide belongs to the drug class benzothiadiazines, simply known as thiazides. Drugs in this class all share a similar dual-ring structure to the thiazide diuretic prototype, chlorothiazide. Chlorthalidone is commonly referred to as a thiazide, but is more properly addressed as a thiazide-type diuretic. Though commonalities exist, chlorthalidone is molecularly unique in structure. ${ }^{18}$ It is also known that chlorthalidone has a longer half-life (40 hours) compared to hydrochlorothiazide (6-9 hours). Some studies found that chlorthalidone concentrates in erythrocytes. The slow release of the drug from these erythrocytes is one explanation for chlorthalidone's long half-life. ${ }^{18}$ A review of available studies suggests that chlorthalidone $25 \mathrm{mg}$ daily is roughly equivalent to hydrochlorothiazide $50 \mathrm{mg}$ daily. This ratio has been found in other analyses as well. ${ }^{18}$

Although no prospective, randomized trials assessing cardiovascular outcomes between the two have been conducted, evidence indicates that chlorthalidone may be superior to hydrochlorothiazide. ${ }^{19}$ The Multiple Risk Factor Intervention Trial (MRFIT) was first to propose a clinical benefit with chlorthalidone compared to hydrochlorothiazide. ${ }^{20}$ MRFIT was a large randomized primary prevention trial assessing the efficacy of a multifactor intervention program, which included dietary advice, smoking cessation counseling, and hypertension treatment compared to usual care (UC). Patients in the study could receive chlorthalidone or hydrochlorothiazide for hypertensive therapy. Approximately 5 years after randomization, the diuretic treatment protocol was changed to replace treatment with hydrochlorothiazide to chlorthalidone due to a $44.1 \%$ higher coronary heart disease (CHD) mortality in clinics predominantly prescribing hydrocholorothiazide compared to the UC group $(P=0.23)$, whereas the clinics predominantly prescribing chlorthalidone had a $-58.2 \%$ (reduction) in CHD mortality compared to UC. ${ }^{21}$ After the change was instituted, the rate of CHD mortality in the previously predominant hydrochlorothiazide clinics decreased to $-7.9 \%$ when compared to UC. ${ }^{21}$

A retrospective, cohort analysis of the MRFIT data was completed to evaluate the cardiovascular endpoints between patients taking the two different drugs. ${ }^{22}$ The results showed a $21 \%$ lower risk of cardiovascular events in patients taking chlorthalidone compared to hydrochlorothiazide (hazard ratio [HR] 0.79 ; 95\% CI: $0.68-0.92 ; P=0.0016$ ). Secondary outcome measures showed that patients prescribed chlorthalidone had a significantly lower SBP, total cholesterol, and low-density lipoprotein (LDL) compared with hydrochlorothiazide. In contrast to these optimistic results, the study also revealed significantly lower serum potassium and higher uric acid with chlorthalidone, but these did not seem to increase events in this group. A small, randomized, prospective study evaluated the efficacy of chlorthalidone to hydrochlorothiazide on ambulatory and trough BP. ${ }^{23}$ This was a force titration study that compared chlorthalidone $25 \mathrm{mg}$ daily to hydrochlorothiazide $50 \mathrm{mg}$ daily, doses which have been shown in other literature to be relatively equivalent. ${ }^{18}$ Though underpowered, the results revealed a trend towards greater reduction in mean SBP with chlorthalidone than with hydrochlorothiazide after 8 weeks $(-12.4$ versus $-7.4 \mathrm{mmHg}$, $P=0.054)$. The difference was attributed to chlorthalidone's greater effect on nighttime SBP ( -13.5 versus $-6.4 \mathrm{mmHg}$; $P=0.009)$. No significant differences in serum potassium were noted between the two groups $(-0.5$ versus $-0.4 \mathrm{mEq} / \mathrm{L}$; $P=0.76) .{ }^{23}$ These trials support the idea that chlorthalidone is a more potent and longer lasting antihypertensive medication compared to hydrochlorothiazide. The MRFIT cohort analysis also supplies additional information regarding its effects on the metabolic profile, which may reveal the mechanism of cardiovascular benefit when compared to hydrochlorothiazide.

\section{Combination therapy versus monotherapy}

Initial combination therapy is recommended for many hypertensive patients requiring a $>20 \mathrm{mmHg}$ decrease in SBP to reach goal. ${ }^{2}$ As seen in the ALLHAT trial, $63 \%$ of the study population was eventually on two or more drugs; and at the end of 5 years only $66 \%$ had achieved controlled BP. ${ }^{8}$ Numerous newer trials have also shown high rates of patients requiring more than one drug for BP management; such as INVEST (80\%), ASCOT-BPLA (78\%), and LIFE (88\%). ${ }^{3,24,25}$ Since a large number of hypertensive patients requires more than one drug to control their hypertension, many trials have examined the benefit of combinations of antihypertensive medications as well as the benefit of combination therapy as initial treatment.

Suggested benefits of combination therapy compared to monotherapy include increased BP control by different mechanisms of action, lower dosing requirements, and fewer adverse events. In a study comparing losartan $50 \mathrm{mg}$ plus barnidipine and losartan $100 \mathrm{mg}$ monotherapy, no significant difference in amount of BP decrease was seen; however there was a higher rate of BP goal obtainment with the combination arm (82.1\% versus $56.0 \%$; $P=0.044) .{ }^{26}$ Both treatment arms were well tolerated. The 
ACCELERATE trial, examined initial combination therapy (aliskiren-amlodipine) compared to initial monotherapy of each component followed by subsequent combination therapy in all arms. ${ }^{27}$ The initial combination therapy had a greater decrease in SBP $(-7.4 \mathrm{mmHg}$ versus aliskiren and $-5.5 \mathrm{mmHg}$ versus amlodipine; $P<0.0001)$ than either monotherapy. After 24 weeks and all patients advanced to combination, the initial monotherapy groups' SBPs decreased and there ceased to be a significant difference between the three arms. This trial also demonstrated balanced adverse events between all groups. ${ }^{27}$

The prompter achievement of BP goal may also be a benefit of initial combination therapy opposed to initial monotherapy later progressing to combination therapy. Results from the VALUE trial, comparing a valsartan-based $\mathrm{BP}$ regimen to a amlodipine-based BP regimen, suggest that obtaining BP control within the first month of treatment is correlated to a lower rate of cardiovascular events. ${ }^{28}$ The previously discussed ACCELERATE trial showed cessation of a significant difference between all combination therapy arms at the end of the study period (24 weeks), yet the SBP in the initial combination treatment arm remained lower than either of the progression groups. ${ }^{27} \mathrm{~A}$ large, case-control study showed an $11 \%$ decrease in cardiovascular events when patients receiving antihypertensive medications were initiated on combination therapy instead of monotherapy. ${ }^{29}$ An important finding in this study was that no difference in events was seen between continuous monotherapy and when monotherapy was switched to combination therapy (Table 2). The only scenario that showed significant decrease in cardiovascular events was the continuous combination therapy arm (odds ratio [OR] 0.74; 95\% CI: 0.65-0.85). These data suggest that the best outcomes may be achieved when combination therapy is initially started in hypertensive patients.

Another advantage to consider with combination therapy is the added benefit of fixed combination drugs.

Table 2 Effects of complete blood pressure therapy (initial + follow-up) on the risk of cardiovascular events

\begin{tabular}{llll}
\hline Initial therapy & $\begin{array}{l}\text { Therapy during } \\
\text { follow-up }\end{array}$ & OR & $\mathbf{9 5 \%} \mathbf{~ C l}$ \\
\hline Monotherapy & Monotherapy & 1.00 & Reference \\
& Combination therapy & 1.00 & 0.91 to I.I0 \\
Combination & Monotherapy & 0.96 & 0.86 to 1.07 \\
therapy & Combination therapy & 0.74 & 0.65 to 0.85 \\
\hline
\end{tabular}

Copyright @ 2011, Reprinted with permission from American Heart Association. Corrao G, Nicotra F, Parodi A, et al. Cardiovascular protection by initial and subsequent combination of antihypertensive drugs in daily life practice. Hypertension. 20II;58(4):566-572. ${ }^{29}$

Abbreviations: $\mathrm{Cl}$, confidence interval; $\mathrm{OR}$, odds ratio.
Fixed combination drugs have the ability to simplify complex medication regimens, increase adherence, and be more cost effective than their separate parts. Most patients require combination therapy for treatment of hypertension, and initiation with a fixed combination drug, such as azilsartan-chlorthalidone, can be clinically beneficial and more convenient.

\section{Conclusion}

Azilsartan-chlorthalidone is a unique combination drug and does have a place in the treatment of hypertension. As the use of ARBs is becoming more popular, azilsartan sets itself apart from the class by demonstrating greater SBP-lowering and longer-lasting hypertensive effects. The chlorthalidone component of the drug also makes this fixed combination distinctive. Chlorthalidone's suggested superiority over hydrochlorothiazide could add to the clinical benefit of this regimen. Additionally a fixed-combination drug can offer the benefit of increasing the amount of patients initiated on combination therapy, leading to patients obtaining their BP goals faster. The effects of azilsartan-chlorthalidone fixed combination have yet to be tested in large, morbidity and mortality trials, but the current data are promising.

\section{Disclosure}

The authors report no conflicts of interest in this work.

\section{References}

1. Roger V, Go A, Lloyd-Jones D. Heart disease and stroke statistics2011 update: a report from the american heart association. Circulation. 2011;123:e18-e209.

2. Chobanian AV, Bakris GL, Black HR, et al. The seventh report of the joint national committee on prevention, detection, evaluation, and treatment of high blood pressure: the JNC 7 report. JAMA. 2003;289(19):2560-2572.

3. Dahlöf B, Devereux RB, Kjeldsen SE, et al; LIFE Study Group. Cardiovascular morbidity and mortality in the losartan intervention for endpoint reduction in hypertension study (LIFE): a randomised trial against atenolol. Lancet. 2002;359(9311):995-1003.

4. Cheung BM, Lau CP, Kumana CR. Combination therapy for hypertension. Hong Kong Med J. 2003;9(3):224-226.

5. Gradman AH, Cutler NR, Davis PJ, Robbins JA, Weiss RJ, Wood BC. Combined enalapril and felodipine extended release (ER) for systemic hypertension. Enalapril-felodipine ER factorial study group. Am J Cardiol. 1997;79(4):431-435.

6. Langtry HD, McClellan KJ. Valsartan/hydrochlorothiazide. Drugs. 1999;57(5):751-755; discussion 756-758.

7. Drugs.com. Top 200 drugs for 2010. Available from: http://www.drugs. com/top200_units.html. Accessed December 13, 2011.

8. Cushman W, Ford C, Cutler J. Success and predictors of blood pressure control in diverse north american settings: the antihypertensive and lipid-lowering treatment to prevent heart attack trial (ALLHAT). J Clin Hypertens. 2002;4:393-404.

9. Goncalves A, Fontes M, Klussmann E, et al. Spinophilin regulates central angiotensin II-mediated effect on blood pressure. J Mol Med. 2011;89:1219-1229. 
10. Chalmers J, Arima H. Management of hypertension: evidence from the blood pressure lowering treatment trialists' collaboration and from major clinical trials. Pol Arch Med Wewn. 2009;119(6):373-380.

11. Edarbi(azilsartan medoxomil) [Package Insert] Deerfield, IL. Takeda Pharmaceuticals America, Inc; 2011.

12. Ojima M, Igata $H$, Tanaka $M$. In vitro antagonistic properties of a new angiotensin type 1 receptor blocker, azilsartan, in receptor binding and function studies. JPET. 2011;336:801-808.

13. Bakris G, Sica D, Weber M. The comparative effects of azilsartan medoxomil and olmesartan on ambulatory and clinic blood pressure. J Clin Hypertens. 2011;13:81-88.

14. White W, Weber M, Sica D. Effects of the angiotensin receptor blocker azilsartan medoxomil versus olmesartan and valsartan on ambulatory and clinic blood pressure in patients with stages 1 and 2 hypertension. Hypertension. 2011;57:413-420.

15. Clinicaltrials.gov. Efficacy and safety of azilsartan medoxomil co-administered with chlorthalidone in participants with essential hypertension. Study NCT00591773. Available from: http:/clinicaltrials. gov/ct2/show/NCT00591773?term=NCT00591773\&rank=1. Accessed December 13, 2011.

16. American Society of Hypertension. Twenty-Sixth Annual Scientific Meeting and Exposition [featured posters, poster 182]. J Clin Hypertens. 2011;13(S1):A12-A163. Available from: http://onlinelibrary.wiley.com/ doi/10.1111/j.1751-7176.2011.00459.x/abstract. Accessed January 2011.

17. American Society of Hypertension. Twenty-Sixth Annual Scientific Meeting and Exposition [featured posters, poster 162]. J Clin Hypertens. 2011;13(S1):A12-A163 Available from: http://onlinelibrary.wiley.com/ doi/10.1111/j.1751-7176.2011.00459.x/abstract. Accessed January 2011

18. Carter B, Ernst M, Cohen J. Hydrochlorothiazide versus chlorthalidone: evidence supporting their interchangeability. Hypertension. 2004;43:4-9.

19. Flack J, Sica D, Nesbitt S. Chlorthalidone versus hydrochlorothiazide as a preferred diuretic: is there a verdict yet? Hypertension. 2011;57:665-666.

20. Five-year findings of the hypertension detection and follow-up program. I. reduction in mortality of persons with high blood pressure, including mild hypertension. Hypertension Detection and Follow-up Program Cooperative Group. JAMA. 1979;242(23):2562-2571.
21. Mortality after $101 / 2$ years for hypertensive participants in the Multiple Risk Factor Intervention trial. Circulation. 1990;82(5): 1616-1628.

22. Dorsch M, Gillespie B, Erikson S. Chlorthalidone reduces cardiovascular events compared with hydrochlorothiazide: a retrospective cohort analysis. Hypertension. 2011;57:689-694.

23. Ernst M, Carter B, Goerdt C. Comparative antihypertensive effects of hydrochlorothiazide and chlorthalidone on ambulatory and office blood pressure. Hypertension. 2006;47:352-358.

24. Pepine CJ, Handberg EM, Cooper-DeHoff RM, et al. A calcium antagonist vs a non-calcium antagonist hypertension treatment strategy for patients with coronary artery disease. the international verapamiltrandolapril study (INVEST): a randomized controlled trial. JAMA. 2003;290(21):2805-2816

25. Dahlof B, Sever P, Poulter N, et al; ASCOT Investigators. Prevention of cardiovascular events with an antihypertensive regimen of amlodipine adding perindopril as required versus atenolol adding bendroflumethiaizde as required in the Ango-Scandinavian Cardiac Outcomes Trial-Blood Pressure Lowering Arm (ASCOT-BPLA): a multicentre randomised controlled trial. Lancet. 2005;366:895-906.

26. Parati G, Giglio A, Lonati L. Effectiveness of barnidipine 10 or $20 \mathrm{mg}$ plus losartan 50-mg combination versus losartan 100-mg monotherapy in patients with essential hypertension not controlled by losartan $50-\mathrm{mg}$ monotherapy: a 12-week, multicenter, randomized, open-label, parallelgroup study. Clin Ther. 2010;32:1270-1284.

27. Brown M, McInnes G, Papst C. Aliskiren and the calcium channel blocker amlodipine combination as an initial treatment strategy for hypertension control (ACCELERATE): a randomized, parallel-group trial. Lancet. 2011;377:312-320.

28. Julius A, Kjeldsen S, Weber M. Outcomes in hypertensive patients at high cardiovascular risk treated with regimens based on valsartan or amlodipine: the VALUE randomised trial. Lancet. 2004;363:2022-2031.

29. Corrao G, Nicotra F, Parodi A, et al. Cardiovascular protection by initial and subsequent combination of antihypertensive drugs in daily life practice. Hypertension. 2011;58(4):566-572.
Vascular Health and Risk Management

\section{Publish your work in this journal}

Vascular Health and Risk Management is an international, peerreviewed journal of therapeutics and risk management, focusing on concise rapid reporting of clinical studies on the processes involved in the maintenance of vascular health; the monitoring, prevention and treatment of vascular disease and its sequelae; and the involvement of

\section{Dovepress}

metabolic disorders, particularly diabetes. This journal is indexed on PubMed Central and MedLine. The manuscript management system is completely online and includes a very quick and fair peer-review system, which is all easy to use. Visit http://www.dovepress.com/ testimonials.php to read real quotes from published authors. 